\title{
Development of planar P-type point contact germanium detectors for low-mass dark matter searches
}

\author{
W.-Z. Wei, H. Mei, K. Kooi, D.-M. Mei ${ }^{\mathrm{a}}$, J. Liu, J.-C. Li, R. Panth, G.-J. Wang \\ Department of Physics, The University of South Dakota, Vermillion, SD 57069, USA
}

Received: 5 May 2021 / Accepted: 23 February 2022 / Published online: 7 March 2022

(C) The Author(s) 2022

\begin{abstract}
The detection of low-energy deposition in the range of sub-eV through ionization using germanium $(\mathrm{Ge})$ with a bandgap of $\sim 0.7 \mathrm{eV}$ requires internal amplification of the charge signal. This can be achieved through high electric field that accelerates charge carriers, which can then generate more charge carriers. The minimum electric field required to generate internal charge amplification is derived for different temperatures. We report the development of a planar point contact Ge detector in terms of its fabrication and the measurements of its leakage current and capacitance as a function of applied bias voltage. With the determination of the measured depletion voltage, the field distribution is calculated using $\mathrm{GeFiCa}$, which predicts that the required electric field for internal charge amplification can be achieved in proximity to the point contact. The energy response to an Am-241 source is characterized and discussed. We conclude that such a detector with internal charge amplification can be used to search for low-mass dark matter.
\end{abstract}

\section{Introduction}

From an experimental point of view, the detection of lowenergy deposition from low-mass dark matter at $\mathrm{MeV}$-scale remains a daunting challenge. Until now, the devices that can meet the energy threshold of $<10 \mathrm{eV}$ required for detecting light dark matter in the $\mathrm{MeV}$-scale range are still in their infancy [1-9].

High-purity Ge (HPGe) detectors are well known for providing excellent energy resolutions over a wide energy range, and thus have been widely used in nuclear and particle physics. A low capacitance Ge detector can be realized with a p-type point contact (PPC) geometry, which allows the detector to achieve the capacitance on the order of $1 \mathrm{pF}$ and can be

\footnotetext{
a e-mail: Dongming.Mei@usd.edu (corresponding author)
}

operated with sub-keV energy threshold [10]. Thus, the PPC Ge detector technology is able to offer both excellent energy resolution and relatively low energy threshold for ultra-low background experiments such as $\mathrm{GeV}$-scale dark matter and axion interactions. This technology has been exploited by two leading ${ }^{76} \mathrm{Ge}$-based neutrinoless double beta decay experiments, the MAJORANA DEMONSTRATOR [11] and GERDA [12]. However, some physical processes requiring an extreme low-energy threshold of $<10 \mathrm{eV}$ such as the detection of $\mathrm{MeV}$-scale dark matter are still unreachable with existing PPC Ge detectors whose energy threshold are hundreds of eV due to the limitation from the read-out electronic noise. Most recently, by the introduction of ultra-low vibration mechanical cooling and wire bonding of a complimentary medaloxide semiconductor (CMOS) charge sensitive preamplifier to a sub-pF PPC Ge detector, Barton et al. [13] have demonstrated electronic noise of $39 \mathrm{eV}-\mathrm{FWHM}$ (full width at half maximum) at $43 \mathrm{~K}$. Thus, $\mathrm{PPC} \mathrm{Ge} \mathrm{detectors} \mathrm{have} \mathrm{great} \mathrm{poten-}$ tial to achieve an extreme low energy threshold if the detectors can be operated at an even lower temperature such as liquid helium temperature $(\sim 4 \mathrm{~K})$ [14].

Traditionally, as shown in Fig. 1, a PPC Ge detector is made of a cylinder of HPGe crystal in which B-implanted $\mathrm{p}^{+}$ and Li-diffused $\mathrm{n}^{+}$electrical contacts are fabricated on the point contact and outer surface contact, respectively. Compared with the traditional PPC Ge detector shown in Fig. 1, a planar PPC Ge detector with amorphous $\mathrm{Ge}$ (a-Ge) contacts, as shown in Fig. 2, can be fabricated much easier due to the two following reasons: (1) four wings in a planar geometry simplify the handling process during the detector fabrication; and (2) fabrication of a-Ge contacts is much simpler than the fabrication of traditional B-implanted $\mathrm{p}^{+}$and Li-diffused $\mathrm{n}^{+}$ electrical contacts $[14,15]$. If a planar PPC detector shown in Fig. 2 is able to achieve a similar detector performance (low detector capacitance and excellent energy resolution) as a conventional PPC detector shown in Fig. 1, it would be more convenient to use a planar PPC detector instead of a cylindri- 


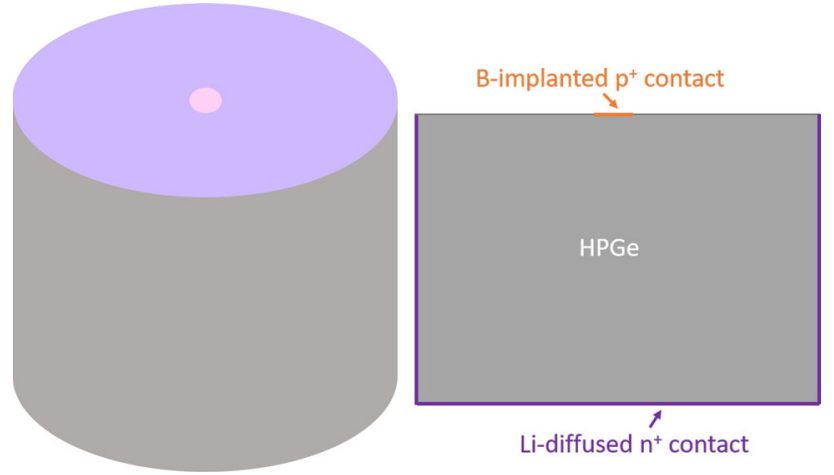

Fig. 1 Cylindrical PPC Ge detector. Left: sketch of the full detector [20]; Right: cross section. The $\mathrm{p}^{+}$contact is shown in orange and the $\mathrm{n}^{+}$contact is shown in purple

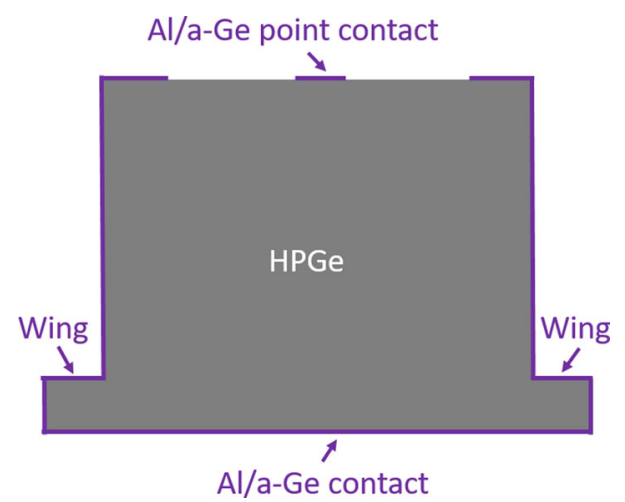

Fig. 2 Cross-sectional drawing of a planar PPC Ge detector. The point and outer surface contacts are in purple and they are both made of a-Ge contact with $\mathrm{Al}$ on the top

cal PPC to explore some interesting physics processes such as charge trapping due to shallow level impurities and charge lost due to deep level impurities in Ge [16]. In addition, it could also be used as a drift detector for medical imaging [17-19].

In order to investigate the possibility of observing internal charge amplification with a planar PPC Ge detector, we have fabricated such a detector in our lab at University of South Dakota (USD). The geometry of the detector is the same as shown in Fig. 2 and the detector was made with the Ge crystal grown by us at USD. The detector has been carefully characterized to examine its performance including leakage current, detector capacitance and energy resolution. Based on the detector performance, we have conducted a numeric calculation to study the electric field distribution inside the detector to find out if the required electric field for internal charge amplification can be achieved with this detector. In this paper, we first show the theoretical requirements for internal charge amplification in Sect. 2. The details about the fabrication and characterization of a custom-build planar PPC Ge detector, and the discussion and the expectation of detector performance for observing internal charge amplifi- cation are presented in Sect. 3. Finally, the conclusions are summarized in Sect. 4.

\section{Theoretical requirements for internal charge amplification}

The initial charge carriers created by external energy deposition in Ge are through ionization and excitation of Ge atoms or impurity atoms in Ge. The ionization and excitation of $\mathrm{Ge}$ atoms to create the secondary charge carriers require a charge carrier to have a minimum kinetic energy of $\sim 0.73 \mathrm{eV}$ (the band gap of $\mathrm{Ge}[1])$ at liquid nitrogen temperature $(77 \mathrm{~K})$. The main remaining impurities in the Ge after zone refining and crystal growth are boron, aluminum, gallium and phosphorus [1]. They are all shallow impurities since their ionization energies are $\sim 0.01 \mathrm{eV}[21]$. Charge carriers or phonons with energies larger than $0.01 \mathrm{eV}$ can easily ionize or excite impurities from the donor level or acceptor level to create charge carriers. Therefore, the minimum energy required to generate a charge carrier and a charged impurity through ionization and excitation of impurities in $\mathrm{Ge}$ is $\sim 0.01 \mathrm{eV}$.

Under an electric field, a charge carrier will be accelerated to gain a kinetic energy of $\Delta E=\frac{1}{2} m^{*} v_{d}^{2}$, where $m^{*}$ is the effective mass of the charge carrier and $v_{d}$ is the drift velocity. During the drifting process, the charge carrier will lose energy by interacting with the crystal lattice which manifests as emitted phonons [22]. These phonons are so-called Neganov-Luke phonons produced by charge transport [23]. As a result, the emission of Neganov-Luke phonons will reduce the kinetic energy of the charge carrier. For a given drifting length, the energy conservation requires the work done on the charge carrier by the externally applied electric field to be equal to the sum of the kinetic energy gained by the charge carrier and the energy loss to the emission of phonons. This is to say that the work done on the charge carrier by the externally applied electric field is always balanced. This energy balance is performed through a competing process between the energy loss to the charge carriers scattering off the orbital electrons and the energy loss to the charge carriers scattering off the lattice [1]. The former will ionize the orbital electrons to create additional charge carriers and the latter will cause the emission of phonons. To obtain internal charge amplification, one prefers the energy loss to the charge carriers scattering off the orbital electrons. This requires the mean scattering length (the mean ionization length) for charge carrier scattering off the orbital electrons to be smaller than the mean scattering length for charge carriers scattering off the lattice.

Under zero electric field, the ionization is a thermal process. Therefore, the mean scattering length depends only on temperature. For charge carriers scattering off the orbital electrons, the mean scattering length $\left(L_{t h}\right)$ can be calculated 


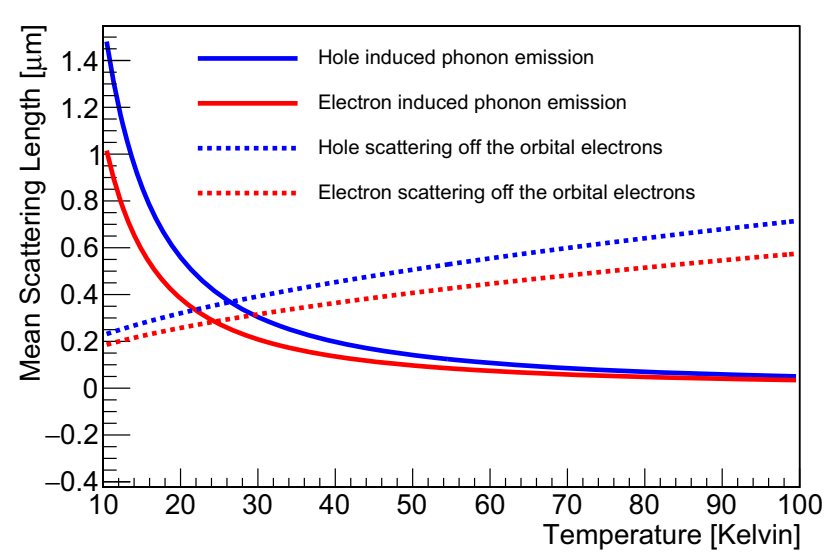

Fig. 3 The mean scattering length versus temperature for charge carriers scattering off the orbital electrons and charge carriers scattering off the lattice, respectively at zero electric field. The solid lines represent hole/electron scattering with the lattice leading to phonon emissions, while the dotted lines stand for scattering with orbital electrons leading to ionization

using $L_{t h}=v_{t h} \times \tau_{t h}$, where $v_{t h}$ is thermal velocity of charge carriers and $\tau_{t h}=m^{*} \frac{\mu_{t h}}{e}$ is the lifetime of charge carriers, with $\mu_{t h}$ the charge mobility. For charge carriers scattering off the lattice, the mean scattering length $\left(\lambda_{t h}\right)$ can be obtained using $\lambda_{t h}=v_{s} \tau_{p h}$, where $v_{s}=5.4 \times 10^{5} \mathrm{~cm} / \mathrm{s}$ is the speed of sound (phonon) in Ge and $\tau_{p h}=m^{*} \mu_{p h} / e$, where $\mu_{p h}=\frac{4.65 \times 10^{5}}{m^{*^{5 / 2}}} \cdot T^{-3 / 2}$ is the charge mobility due to acoustic deformation potential scattering [24] and $T$ is temperature. Figure 3 shows the mean scattering length as a function of temperature for ionization and phonon emission, respectively.

It is clear that the mean scattering length for charge carriers scattering off the orbital electrons is smaller than that of charge carriers scattering off the lattice at temperature less than $30 \mathrm{~K}$. To effectively generate more charge carriers with internal charge amplification, one would prefer to run the detector at a temperature less than $30 \mathrm{~K}$.

For drifting charge carriers under the electric field, the work done on a charge carrier can be expressed as:

$\mathbf{F} \cdot \mathbf{L}=e \mathbf{E} \cdot \mathbf{L}=e E L$

where $F$ is the electric force, $L$ is the drifting path length along the direction of electric force, and $\mathbf{E}$ is the electric field. For one interaction length, $L$ is equal to the mean scattering length for charge carriers scattering off the orbital electrons, that is

$L=v_{d} \cdot \tau=\mu E \frac{m^{*} \mu}{e}=\mu^{2} E \frac{m^{*}}{e}$,

where $\tau$ is the relaxation time of a charge carrier and $\mu$ is the drift mobility.

For temperature less than $30 \mathrm{~K}$, the mean scattering length for charge carriers scattering off the orbital electrons is smaller than that for charge carriers scattering off the lattice, as shown in Fig. 3. Therefore, the work done on a charge carrier by the applied electric field will mainly accelerate the charge carrier to gain sufficient kinetic energy and hence generate more e-h pairs through internal charge amplification. The minimum electric field required to trigger internal charge amplification can be roughly estimated using Eq. (1). Since the minimum ionization energy of Ge atoms is $\sim 0.73 \mathrm{eV}$ (the band gap energy of $\mathrm{Ge}$ ) and the average ionization length is about $0.4 \mu \mathrm{m}$ at zero electric field as shown in Fig. 3, the minimum electric field required is just under $2 \times 10^{4} \mathrm{~V} / \mathrm{cm}$. Similarly, the average ionization energy for shallow impurity atoms in $\mathrm{Ge}$ is about $0.01 \mathrm{eV}$. Thus, the minimum electric field required to trigger internal charge amplification through ionization of impurity atoms in Ge is roughly estimated to be just above $1.0 \times 10^{4} \mathrm{~V} / \mathrm{cm}$. It is worth mentioning that if the temperature is significantly lower than $30 \mathrm{~K}$, the average ionization length under an applied electric field can be significantly larger than what is derived here and shown in Fig. 3. Therefore, the minimum electric field required to trigger internal charge amplification can be significantly lower than what is estimated for both ionization of Ge atoms and ionization of impurity atoms. However, the detailed discussion requires sophisticate models in calculating the average ionization length and is beyond the scope of this work.

For temperature greater than $30 \mathrm{~K}$, the average ionization length is larger than the mean scattering length for charge carriers scattering off the lattice. Hence, it is likely there will be energy loss to the emission of phonons before kicking off the internal charge amplification. Thus, energy conservation requires the work done by the electric field be equal to the sum of the gained kinetic energy and the energy of emitted phonons. Therefore, the minimum electric field required to generate an e-h pair on Ge atoms or impurity atoms during a drifting process with a drifting length of $L$ can be calculated from the following relation:

$e E \times L=\Delta E+\frac{L}{\lambda_{p h}} \times \Delta \bar{E}_{p h}$,

where $\lambda_{p h}=v_{s} \tau$ is the mean scattering length for producing phonons, $\Delta \bar{E}_{p h}$ is the average energy of emitted phonons, which is estimated as $\Delta \bar{E}_{p h}=\frac{h v_{s}}{a}=0.04 \mathrm{eV}$, where $h$ is the Planck constant and $a=0.56 \mathrm{~nm}$ is the lattice constant of Ge.

Combining Eqs. (2) and (3), we have

$e E \times \mu^{2} E \frac{m^{*}}{e}=\Delta E+\frac{\mu E}{v_{s}} \cdot \Delta \bar{E}_{p h}$.

Rearranging the terms in Eq. (4), we have

$m^{*} \mu^{2} E^{2}-\frac{\mu}{v_{s}} \Delta \bar{E}_{p h} E-\Delta E=0$.

For this quadratic equation, we have $E=\frac{-b \pm \sqrt{b^{2}-4 a c}}{2 a}$, where $a=m^{*} \mu^{2}, b=-\frac{\mu}{v_{s}} \Delta \bar{E}_{p h}$, and $c=-\Delta E$ is the 


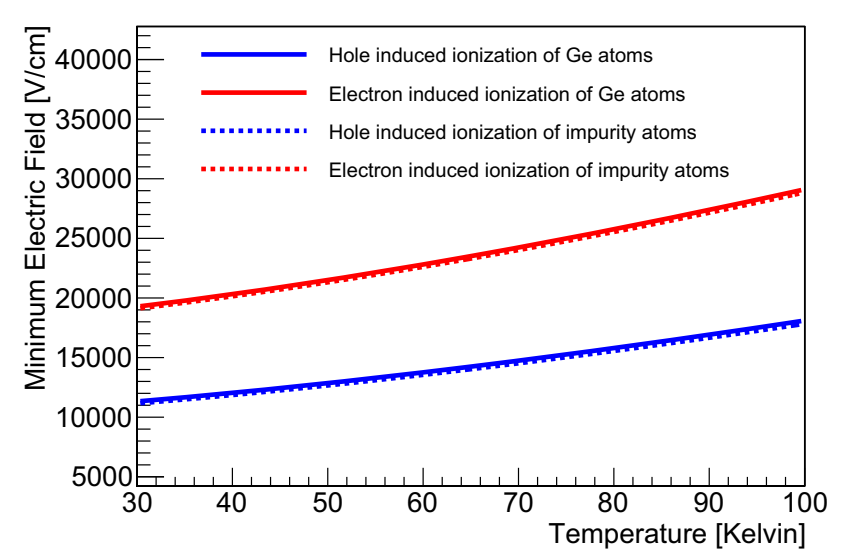

Fig. 4 The minimum electric field required for internal charge amplification of $\mathrm{Ge}$ atoms and impurity atoms in a temperature range from 30 to $100 \mathrm{~K}$

energy required to generate an e-h pair in $\mathrm{Ge}, \sim 0.73 \mathrm{eV}$, at liquid nitrogen temperature [25].

Similarly, energy conservation during this process can be used to calculate the minimum electric field required to generate an e-h pair on impurity atoms in Ge using Eq. (4). However, now $\Delta E$ is the ionization energy of shallow impurities in $\mathrm{Ge}(\Delta E \geq 0.01 \mathrm{eV})$.

When the temperature is above $30 \mathrm{~K}$, the charge drift mobility in Ge is dominated by the contribution from phonon scattering mobility and neutral impurity scattering [24]. The Hall mobility for holes is often taken as $\mu_{h 0}=42,000$ $\mathrm{cm}^{2} /(\mathrm{V} \cdot \mathrm{s})$ and the Hall mobility for electrons is $\mu_{e 0}=36,000$ $\mathrm{cm}^{2} /(\mathrm{V} \cdot \mathrm{s})$ for Ge according to IEEE standard [26]. The relationship between the Hall mobility and the drift mobility is given by $\mu_{h}=\mu_{h 0} / 1.03$ for holes and $\mu_{e}=\mu_{e 0} / 0.83$ for electrons, respectively [26]. Utilizing Eq. (5) with $m^{*}=0.21 \mathrm{~m}_{0}$ [1] for holes and $m^{*}=0.12 \mathrm{~m}_{0}$ [1] for electrons, where $\mathrm{m}_{0}=$ $511,000 \mathrm{eV} / \mathrm{c}^{2}$ is the electron mass in vacuum, we obtain the minimum electric field as a function of temperature from 30 $\mathrm{K}$ to $100 \mathrm{~K}$ as shown in Fig. 4.

It is clear that the minimum field required to generate an e-h pair in $\mathrm{Ge}$ is above $10^{4} \mathrm{~V} / \mathrm{cm}$. There is a large difference in the minimum required field for generating more e-h pairs when drifting electrons and holes across the detector. A weak temperature dependence is also seen in Fig. 4. It shows that the minimum field required to generate more e-h pairs decreases as temperature decreases. This indicates that an internal charge amplification can be achieved at a lower electric field when the detector is operated at a lower temperature. At liquid nitrogen temperature $(77 \mathrm{~K})$, the required electric field for internal charge amplification must be obtained through a special geometry design at which high electric field is achievable in a designated detector region. We studied this hypothesis using a customized planar PPC detector.
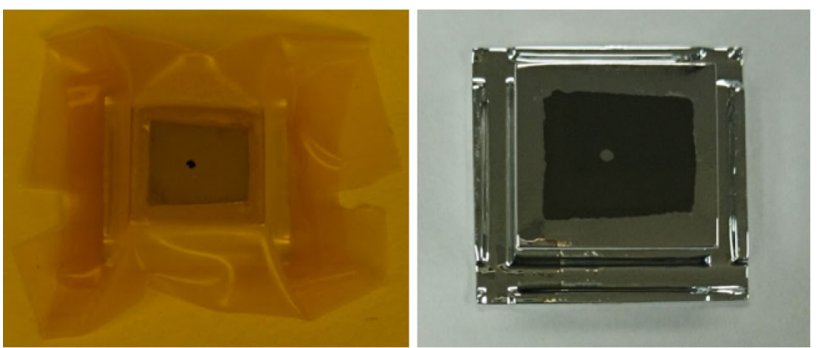

Fig. 5 Left: the detector was covered with acid resistant tape and paint (picine) to form the outer contact and point contact, respectively. Right: the top view of the completed planar PPC Ge detector, USD-W06. The uneven edge of the outer contact was caused by cutting the acid resistant tape manually

\section{Operation and characterization of a custom-build planar PPC detector}

\subsection{Detector fabrication process}

To convert an HPGe crystal into a planar PPC detector, the fabrication process of depositing a-Ge and $\mathrm{Al}$ on all the detector surfaces is the same as in fabricating a planar detector from an HPGe crystal. Please refer to our previous work $[25,27]$ for the fabrication details. After the electric contacts (a-Ge and Al) were deposited, as shown in Fig. 5(left), an acid-resistant tape was used to cover the bottom, sides and a portion of the top surface to create the outer contact of the PPC detector. To form the point contact, an etch resistant paint (picine), as shown in Fig. 5(left), was applied in the center of the top surface. The exposed $\mathrm{Al}$ on the point contact face was then removed using a diluted HF dip (100:1). The tape and paint were removed and the detector fabrication was completed. Figure 5(right) shows the top view of the completed planar PPC detector, USD-W06, fabricated at USD. The diameter of the point contact is around $0.6 \mathrm{~mm}$ and the thickness of the detector is $9.4 \mathrm{~mm}$. The width of the outer contact on the top surface of the crystal is around $6.0 \mathrm{~mm}$. Other dimensions of this detector are presented in Table 1.

\subsection{Detector characterization}

After the detector was fabricated, it was loaded in a variabletemperature cryostat to conduct electrical and spectroscopy

Table 1 The dimensions of the planar PPC Ge detector, USD-W06, fabricated at USD in this work

\begin{tabular}{lll}
\hline & Length $(\mathrm{cm})$ & Width $(\mathrm{cm})$ \\
\hline Crystal top & 1.37 & 1.37 \\
Crystal bottom & 2.12 & 2.08 \\
\hline
\end{tabular}




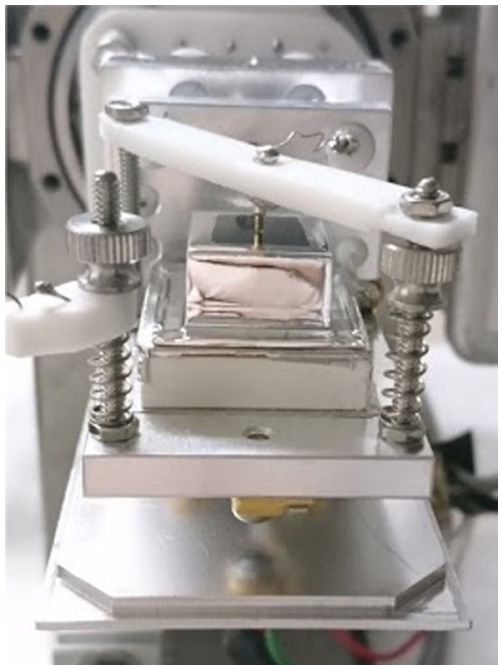

Fig. 6 Shown is the detector USD-W06 loaded in a variabletemperature cryostat for characterization

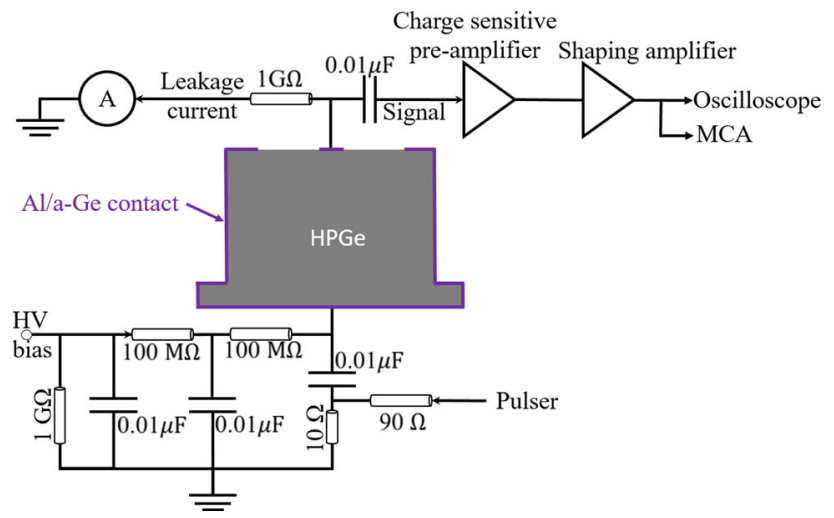

Fig. 7 Electronic circuit for detector characterization

measurements, as shown in Fig. 6. This cryostat is provided by the Lawrence Berkeley National Laboratory (LBNL). Figure 7 shows the electronics used for signal processing and conducting electrical and spectroscopy measurements in this work.

\subsubsection{Electrical measurements}

The following two electrical measurements were conducted in this study: (1) the leakage current versus the applied voltage at $79 \mathrm{~K}$; and (2) the detector capacitance as a function of the applied voltage at $79 \mathrm{~K}$. For both electrical measurements, a positive bias voltage was applied to the bottom contact of the detector, USD-W06, and the signals were read out from the top point contact.

Figures 8 and 9 present the measured leakage current and the detector capacitance as a function of the detector bias voltage at $79 \mathrm{~K}$. As shown in Fig. 9, detector USD-W06 is fully depleted at $60 \mathrm{~V}$ with leakage current at around $0.5 \mathrm{pA}$

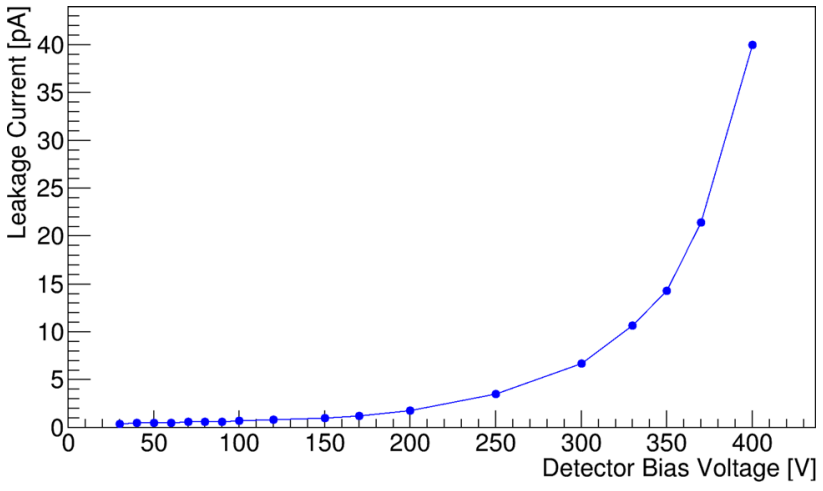

Fig. 8 Measured leakage current as a function of bias voltage at $79 \mathrm{~K}$ for detector USD-W06. Low leakage current $(\sim 0.5 \mathrm{pA})$ at full depletion voltage $(60 \mathrm{~V})$ shows the good quality of a-Ge contacts

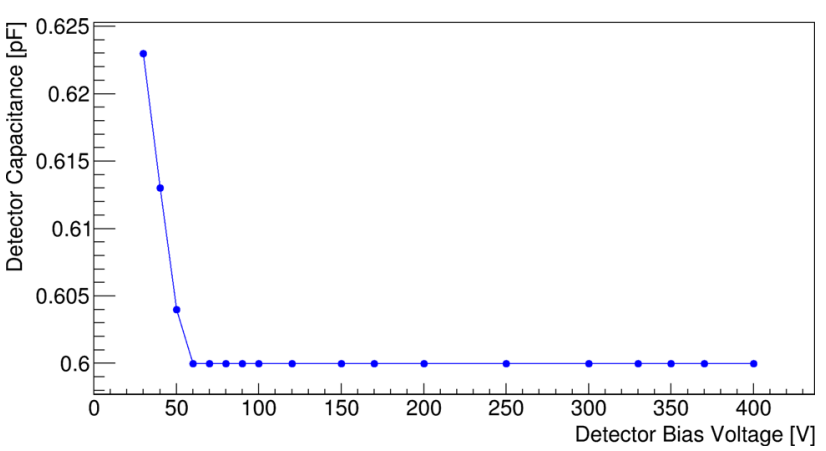

Fig. 9 Measured detector capacitance as a function of bias voltage at $79 \mathrm{~K}$ for detector USD-W06. The full depletion voltage of the detector was determined to be $60 \mathrm{~V}$ since the detector capacitance became a constant after the detector was fully depleted

from Fig. 8. Also, as shown in Fig. 9, this planar PPC detector is able to reach a low capacitance of $0.6 \mathrm{pF}$. Since the signal to background is anti-proportional to the detector capacitance, a lower capacitance will enhance the signal to background ratio and hence has the potential to become a low-threshold detector if operated at an even lower temperature such as liquid helium temperature.

\subsubsection{Distributions of electrical field and potential inside the detector}

From Sect. 2, the required electric field for the internal charge amplification to occur is at least $\sim 15,000 \mathrm{~V} / \mathrm{cm}$ at $79 \mathrm{~K}$. To find out the minimum applied voltage to allow our detector to achieve this required electric field, we have investigated the electric field distribution inside the detector using GeFiCa (Germanium Field Calculator) [28], which was created to demonstrate analytic and numeric methods to calculate static electric fields and potentials in HPGe detectors. A planar geometry with the same dimensions as the real detector (except four wings where the electric field is expected to be extremely low) has been built in GeFiCa. As shown in 


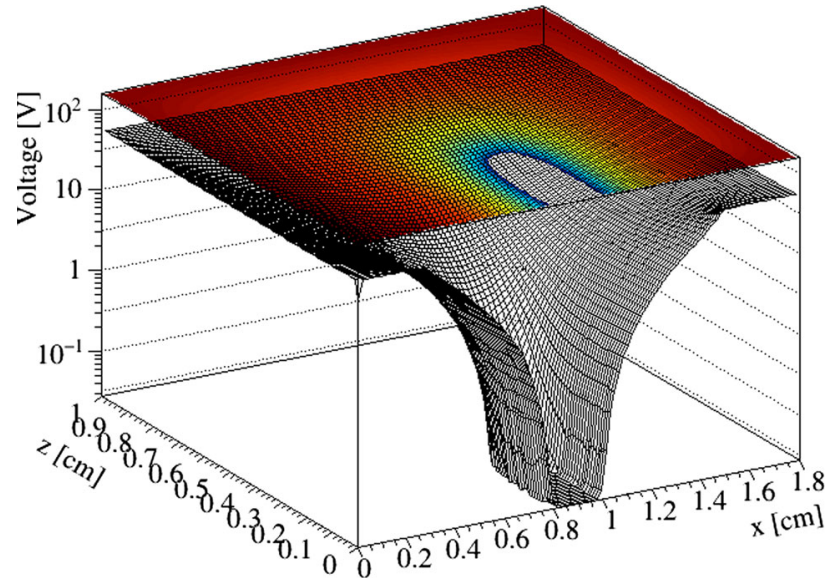

Fig. 10 The potential field distribution in the detector when a positive voltage of $55 \mathrm{~V}$ was applied to the outside surface contact. The white area indicates that the detector is not fully depleted

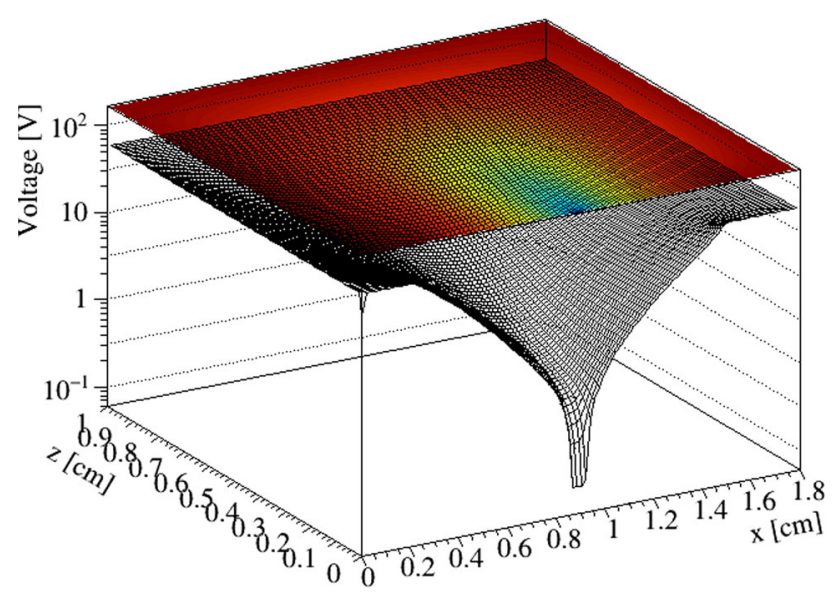

Fig. 11 The potential field distribution in the detector when a positive voltage of $60 \mathrm{~V}$ was applied to the outside surface contact.The absence of any white area indicates that the detector is fully depleted

Figs. 10 and 11, the detector can be fully depleted at $60 \mathrm{~V}$ in agreement with the measurement as shown in Fig. 9. Given the inputs for the detector dimensions and depletion voltage $(60 \mathrm{~V})$, the impurity concentration of the crystal predicted by GeFiCa is $3.3 \times 10^{9} / \mathrm{cm}^{3}$. This is close to the measured impurity concentration of the two planar detectors (USD-W04 and USD-RL01) [29] made from the same crystal as the detector, USD-W06, in this work. Figures 12 and 13 show the distributions of the electric field and potential inside the detector with an applied voltage of $400 \mathrm{~V}$ on the outside surface contact. As shown in Fig. 12, the maximum electric field in the detector at $400 \mathrm{~V}$ is between $8000 \mathrm{~V} / \mathrm{cm}$ and $9000 \mathrm{~V} / \mathrm{cm}$, which is significantly lower than the required minimum electric field of $\sim 15,000 \mathrm{~V} / \mathrm{cm}$ for internal charge amplification to occur inside the detector. This indicates that no internal charge amplification can be observed in our detector with an applied voltage of $400 \mathrm{~V}$. Through further modeling in GeFiCa, we

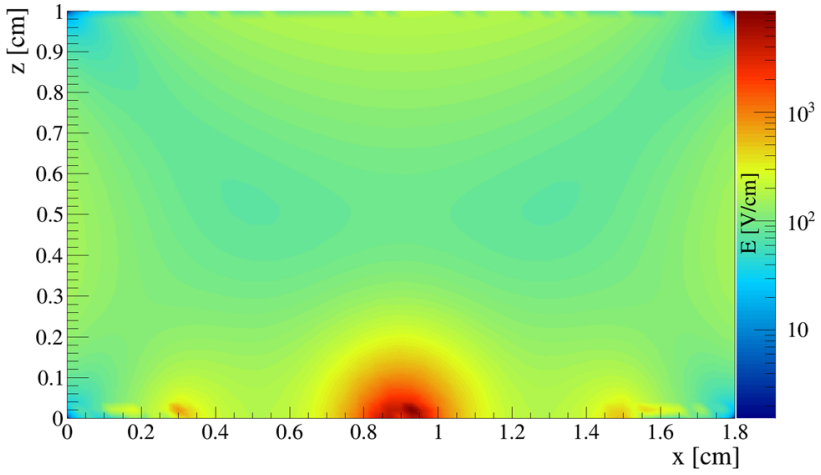

Fig. 12 The electric field distribution in the detector when a positive voltage of $400 \mathrm{~V}$ was applied to the outside surface contact

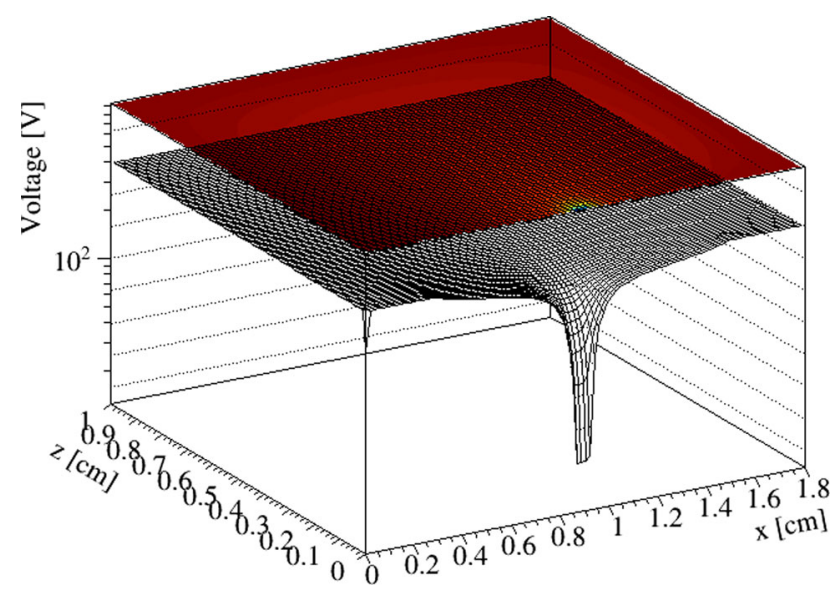

Fig. 13 The potential field distribution in the detector when a positive voltage of $400 \mathrm{~V}$ was applied to the outside surface contact

found that $900 \mathrm{~V}$ is required to achieve the required electric field, as shown in Fig. 14. Unfortunately, due to the fact that the leakage current of our detector jumped to a very high value starting from around $420 \mathrm{~V}$ because of field penetration into a-Ge, we were unable to apply the bias voltage beyond $400 \mathrm{~V}$.

\subsubsection{Spectroscopy measurements}

A radioactive source, Am-241, was used to conduct the spectroscopy measurements in order to: (1) find out if the internal charge amplification will not occur in the detector at $400 \mathrm{~V}$ as predicated by $\mathrm{GeFiCa}$; and (2) examine the detector energy resolution.

According to the discussion in Sect. 2, if the internal charge amplification occurs in the spectroscopy measurements with Am-241, the energy peaks are expected to shift significantly as a function of applied voltage. Unlike a fraction of charge increasing due to the improvement of the charge collection efficiency as a function of electric field, the internal charge amplification multiplies charge produc- 


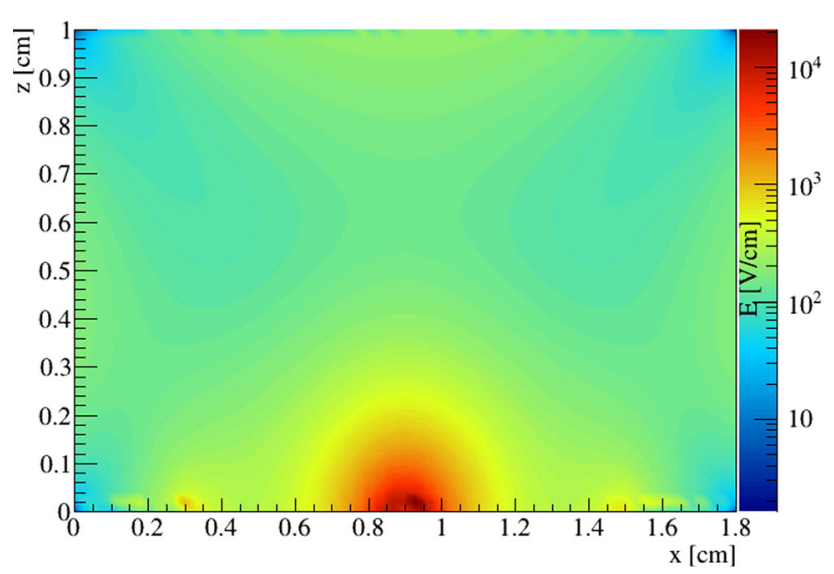

Fig. 14 The electric field distribution in the detector when a positive voltage of $900 \mathrm{~V}$ was applied to the outside surface contact

tion by more than a factor of 2 or higher depending on the applied electric field. This multiplication of charge production can significantly shift the peak positions.

In this study, there are two setups for the collection of the energy spectrum of Am-241: (1) the source was placed on the top of the cryostat, facing the top side of the detector; and (2) the source was placed at the bottom of the cryostat, facing the bottom side of the detector. For each setup, the energy spectra of Am-241 at $79 \mathrm{~K}$ were acquired with positive bias voltages of $200 \mathrm{~V}, 300 \mathrm{~V}$ and $400 \mathrm{~V}$ applied to the detector bottom with the detector configuration shown in Fig. 6. The signals were read out from the point contact using a charge sensitive pre-amplifier operated at room temperature followed by an ORTEC 671 shaping amplifier set to an optimized shaping time of $2 \mu \mathrm{s}$. Figure 15 shows the energy spectra of Am241 taken at three voltages with the source placed at the two positions mentioned above. The data taking times for each of the applied voltages were $1.5 \mathrm{~h}$ when the source was placed on the top of the cryostat, while the data taking times were $14.5 \mathrm{~h}, 9 \mathrm{~h}$ and $8 \mathrm{~h}$ for the applied bias voltages of $200 \mathrm{~V}, 300$

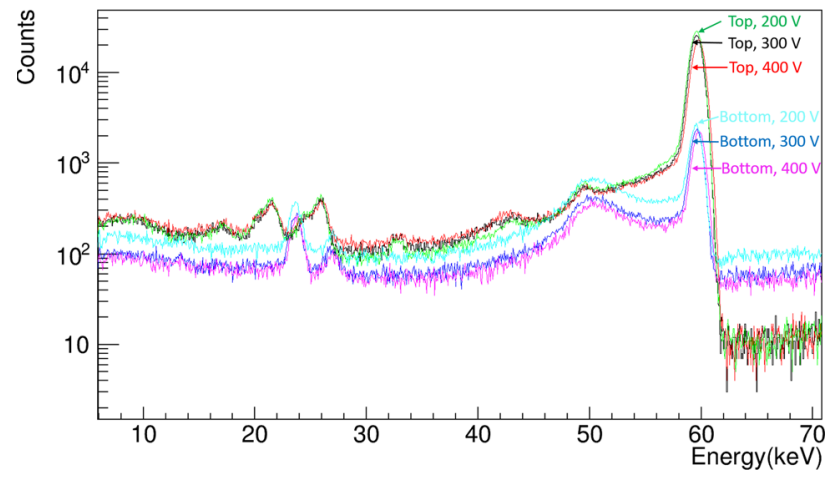

Fig. 15 Energy spectra of a Am-241 source measured with the detector USD-W06. The spectra were taken at three voltages, $200 \mathrm{~V}, 300 \mathrm{~V}$ and $400 \mathrm{~V}$ with the source placed at the top and the bottom of the cryostat for each voltage

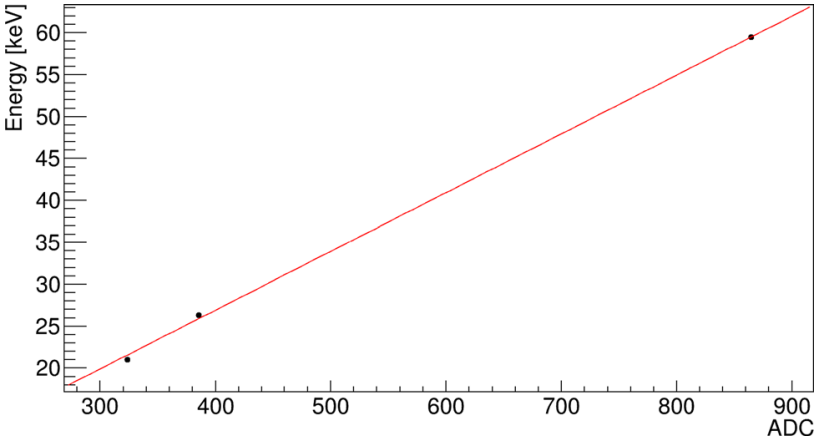

Fig. 16 Converting the ADC channel number to the energy with linear calibration function of $E=p 0+p 1 \cdot A$, where $E$ is the energy in $\mathrm{keV}$, $A$ is the ADC channel number, and $p 0$ and $p 1$ are the fitting parameters with $p 0$ the intercept in $\mathrm{keV}$ and $p 1$ the slope

$\mathrm{V}$ and $400 \mathrm{~V}$, respectively, when the source was placed at the bottom of the cryostat. The $\mathrm{x}$-axis is the energy in $\mathrm{keV}$, which is converted from the ADC count using three photon peaks from Am-241, $20.8 \mathrm{keV}, 26.3 \mathrm{keV}$ and $59.5 \mathrm{keV}$ [30], with the applied voltage at $200 \mathrm{~V}$ and the source placed at the top of the cryostat. The calibration curve is shown in Fig. 16. The linear fit function is: $E=p 0+p 1 \cdot A$, where $E$ is the energy in $\mathrm{keV}$, $\mathrm{A}$ is the $\mathrm{ADC}$ channel number, $p 0=-1.104 \pm 1.109$ $\times 10^{-4} \mathrm{keV}$ is the intercept and $p 1=0.0701 \pm 1.283 \times 10^{-7}$ is the slope.

As shown in Fig. 15, for both positions of the source, no evident energy position shift has been observed for each energy peak. This confirms that the charge internal amplification didn't take place in the detector with applied voltage at $400 \mathrm{~V}$. For our future work, we will optimize our fabrication process to improve the quality of the a-Ge contact so that it will allow the detector to withstand the required electric field to observe the internal charge amplification at $79 \mathrm{~K}$.

To examine the detector energy resolution, it is necessary to identify each energy peak measured from Am-241. From Fig. 15 we can only see that the resolution in the region of $\sim 20 \mathrm{keV}$ is worse than the $59.5 \mathrm{keV}$ peak. From atomic data we know that for high- $\mathrm{Z}$ atoms, we expect clusters of $\mathrm{X}$-rays. In addition, the energy region of $26.3 \mathrm{keV}$, a $\gamma$ ray from Am241 source is contaminated by several $\mathrm{X}$-rays from the $\sim 20$ $\mathrm{keV}$ region, which are very close in energy. Therefore, the two peaks were not well resolved. It is worth mentioning that there are more materials between the source and the detector when the source was placed at the bottom of the cryostat compared to that of the source at the top. Consequently, several low-energy X-rays and $\gamma$ rays $(\sim 20.8 \mathrm{keV}$ and 26.3 $\mathrm{keV}$ ) seen with the source at the top were not seen when the source was placed at the bottom. Instead, we observed two emitted X-rays with energy $24 \mathrm{keV}$ and $27.5 \mathrm{keV}$, as shown in Fig. 15, from indium due to the process of fluorescence when $59.5 \mathrm{keV} \mathrm{X}$-ray interacted with the indium foil which is set underneath of the detector on the inside of the 


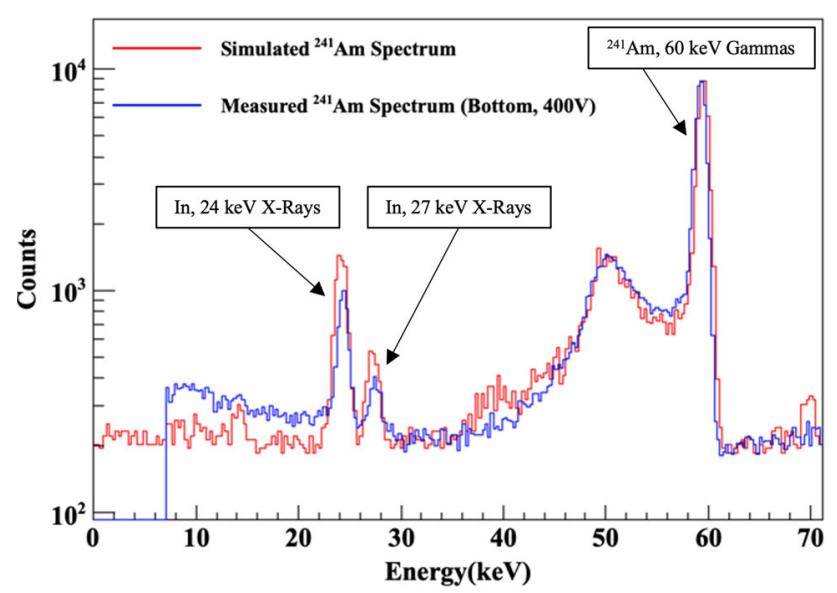

Fig. 17 A comparison between the Monte Carlo simulation and the measurement when the source was placed at the bottom. The blue line is the measured energy spectrum and the red line is the simulated energy spectrum. The simulation confirms the fluorescent emission of X-rays (24 keV and $27.5 \mathrm{keV}$ ) from the inner material of indium

cryostat. To confirm the fluorescent emission of X-rays from the inner material of indium, we conducted a Geant4-based Monte Carlo simulation [31] for the source at the bottom with a detailed geometry of the cryostat based on our best understanding. Figure 17 shows a comparison between the measurement and the simulation when the source is placed at the bottom. It was clear that two distinct X-rays observed in Fig. 15 when the source was placed at the bottom were from the fluorescent emission of indium. These two distinct $\mathrm{X}$-rays provide a good opportunity to examine the detector energy resolution for low-energy X-rays with energy below $59.5 \mathrm{keV}$.

To obtain the detector energy resolution, the two low energy peaks at $24 \mathrm{keV}$ and $27.5 \mathrm{keV}$ were fitted together using two standard Gaussian distributions and a linear background distribution with $\chi^{2} / \mathrm{ndf}=115.7 / 120$ :

$p 0 \cdot e^{\left[-\frac{1}{2}\left(\frac{E-p 1}{p 2}\right)^{2}\right]}+p 3 \cdot e^{\left[-\frac{1}{2}\left(\frac{E-p 4}{p 5}\right)^{2}\right]}+p 6+p 7 \cdot E$,

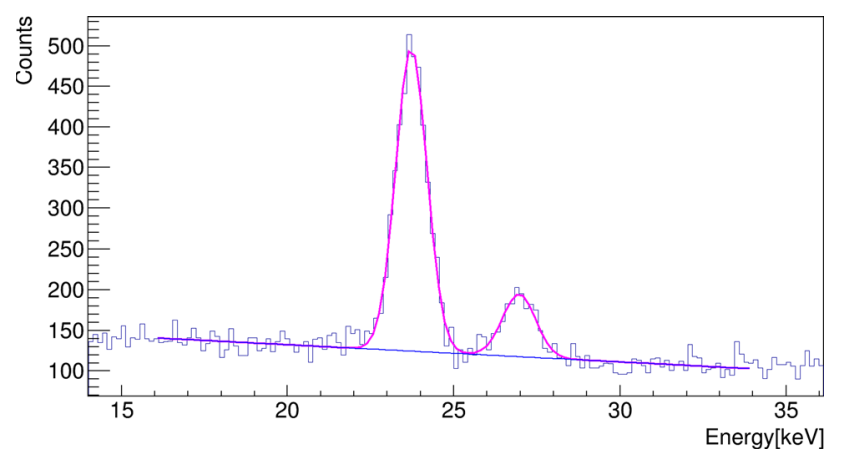

Fig. 18 The fitted energy peaks at $24 \mathrm{keV}$ and $\sim 27.5 \mathrm{keV}$ from fluorescent emission of X-ray with applied voltage at $400 \mathrm{~V}$ when the source was placed at the bottom of the cryostat

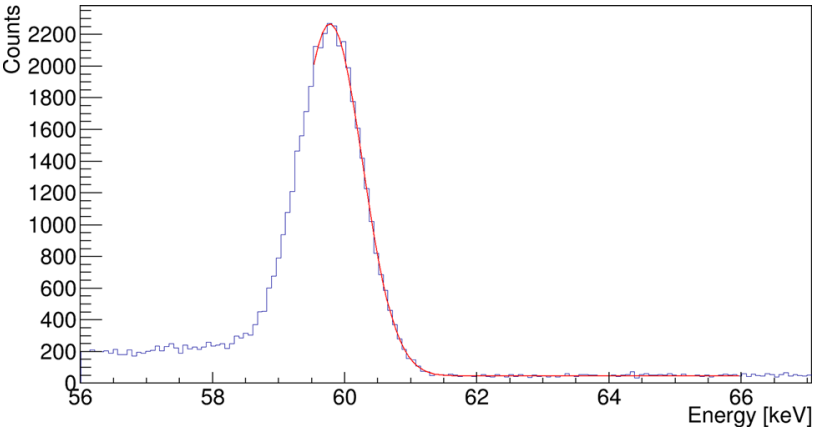

Fig. 19 The fitted energy peak at $59.5 \mathrm{keV}$ from Am-241 with applied voltage at $400 \mathrm{~V}$ when the source was placed at the bottom of the cryostat

where $p 0=373 \pm 10.4$ and $p 3=77.01 \pm 6.81$ are the normalization constants, $p 1=23.72 \pm 0.01 \mathrm{keV}$ and $p 4=26.96 \pm$ $0.05 \mathrm{keV}$ are the center values for the energy peaks at $24 \mathrm{keV}$ and $27.5 \mathrm{keV}$, respectively, $p 2=0.4707 \pm 0.0117 \mathrm{keV}$ and $p 5=0.4976 \pm 0.0497 \mathrm{keV}$ are the Gaussian widths (sigma) for the energy peaks at $24 \mathrm{keV}$ and $27.5 \mathrm{keV}$, respectively, $p 6$ $=174.6 \pm 5.0$ is a constant, $p 7=-2.114 \pm 0.19$ is the slope, and $E$ is the energy in $\mathrm{keV}$. The energy peak at $59.5 \mathrm{keV}$ was partially fitted using a standard Gaussian distribution with $\chi^{2} / \mathrm{ndf}=85.38 / 89, p 0 \cdot e^{\left[-\frac{1}{2}\left(\frac{E-p 1}{p 2}\right)^{2}\right]}$, with $p 0=2222 \pm 16.9$ (normalization constant), $p 1=59.78 \mathrm{keV}$ (center value), and $p 2=0.4935 \pm 0.0055 \mathrm{keV}$ (Gaussian width). This is because the background on the left side is higher than the right side and we are only interested in knowing the energy resolution. As examples, Figs. 18 and 19 show the fittings of the energy peaks at $24 \mathrm{keV}, 27.5 \mathrm{keV}$ and $59.5 \mathrm{keV}$ at $400 \mathrm{~V}$ when the radiation source was placed at the bottom of the cryostat. Figure 20 is a fitted artificial peak due to the injected pulses from the high voltage line in order to obtain the noise level. The electronic circuit presented in Fig. 7 shows how the pulse was generated to measure the electronic noise of the detector. The peak in Fig. 20 was fitted using a standard Gaussian

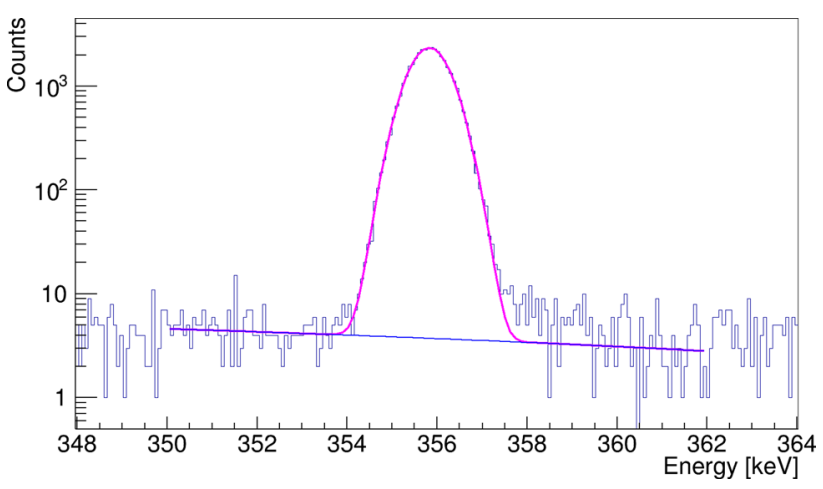

Fig. 20 The fitted artificial peak due to the injected pulses from the high voltage line with applied voltage at $400 \mathrm{~V}$ when the source was placed at the bottom of the cryostat 
Table 2 The FWHM of three energy peaks from Am-241 and the pulser peak at $400 \mathrm{~V}$ when the source was placed at the bottom of the cryostat. The energies presented here are the expected values. For each energy peak, the relative statistic driven energy resolution was determined by subtracting in quadrature the FWHM at the pulser from the FWHM at the energy peak and then divided by the energy

\begin{tabular}{llll}
\hline $\begin{array}{l}\text { Energy } \\
\text { Peak }(\mathrm{keV})\end{array}$ & $\begin{array}{l}\text { FWHM at the } \\
\text { energy peak } \\
(\mathrm{keV})\end{array}$ & $\begin{array}{l}\text { FWHM at the } \\
\text { pulser }(\mathrm{keV})\end{array}$ & $\begin{array}{l}\text { Relative statistic } \\
\text { driven energy res- } \\
\text { olution }(\%)\end{array}$ \\
\hline 24.0 & 1.11 & 1.06 & 1.37 \\
27.5 & 1.17 & 1.06 & 1.80 \\
59.5 & 1.16 & 1.06 & 0.79 \\
\hline
\end{tabular}

distribution and linear background distribution with $\chi^{2} / \mathrm{ndf}$ $=199.9 / 164$ :

$p 0 \cdot e^{\left[-\frac{1}{2}\left(\frac{E-p 1}{p^{2}}\right)^{2}\right]}+p 3+p 4 \cdot E$,

where $p 0, p 1, p 2$ and $E$ were defined in Eq. (6), $p 3$ is a constant and $p 4$ is the slope. The best fit results for these parameters are: $p 0=2346 \pm 15.0, p 1=355.8 \pm 0.0 \mathrm{keV}, p 2=0.4504$ $\pm 0.0017 \mathrm{keV}, p 3=57.24 \pm 14.71$, and $p 4=-0.1504 \pm$ 0.0412 . Table 2 summarizes the full width at half maximum (FWHM) of the three energy peaks and the pulser peak shown in Figs. 18, 19 and 20. Also shown in Table 2 are the statistically driven energy resolutions for each peak. These are determined by subtracting in quadrature the FWHM of the injected pulse peak from the FWHM of the respective energy peaks.

\section{Conclusions}

We have experimentally shown that a planar PPC detector can be fabricated successfully using USD-grown crystals with aGe contacts. The minimum required electric field to achieve internal charge amplification is derived using the work done on the charge carriers by the externally applied electric field. This work done must be equal to the sum of the kinetic energy gained by the charge carriers and the energy loss to the emission of photons. This relation is required by the energy conservation during the drifting process. From the calculation using GeFiCa, we find that the planar PPC Ge detector can be used to achieve the high electric field needed for triggering internal charge amplification. Such a detector, when operated at a bias voltage of higher than $900 \mathrm{~V}$, will generate a field strength that is higher than $15,000 \mathrm{~V} / \mathrm{cm}$ near the point contact. This high field will then amplify charge carriers to generate more charge carriers. We examined the energy resolution of the planar PPC detector and found that a good energy resolution can be achieved with the current electronics as shown in Table 2. If a low-noise electronic system is adapted and the energy resolution is dominated by the sta- tistical variation, the energy resolution of $1.37 \%$ at $24 \mathrm{keV}$, $1.80 \%$ at $27.5 \mathrm{keV}$, and $0.79 \%$ at $59.5 \mathrm{keV}$ can be achieved. If internal charge amplification can be realized by this detector, which allows us to measure single electron-hole pair, this planar PPC detector can be used for low-mass dark matter searches.

Acknowledgements The authors would like to thank Mark Amman for his instructions on fabricating planar detectors, and the Nuclear Science Division at Lawrence Berkeley National Laboratory for providing the vacuum cryostat. We would also like to thank Christina Keller for a careful reading of this manuscript. This work was supported in part by NSF NSF OISE 1743790, DOE grant DE-FG02-10ER46709, DESC0004768, the Office of Research at the University of South Dakota and a research center supported by the State of South Dakota. Computations supporting this project were performed on High Performance Computing systems at the University of South Dakota, funded by NSF Award OAC-1626516.

Data Availability Statement This manuscript has no associated data or the data will not be deposited. [Authors' comment: All data has been used and included in the paper.]

Open Access This article is licensed under a Creative Commons Attribution 4.0 International License, which permits use, sharing, adaptation, distribution and reproduction in any medium or format, as long as you give appropriate credit to the original author(s) and the source, provide a link to the Creative Commons licence, and indicate if changes were made. The images or other third party material in this article are included in the article's Creative Commons licence, unless indicated otherwise in a credit line to the material. If material is not included in the article's Creative Commons licence and your intended use is not permitted by statutory regulation or exceeds the permitted use, you will need to obtain permission directly from the copyright holder. To view a copy of this licence, visit http://creativecomm ons.org/licenses/by/4.0/.

Funded by $\mathrm{SCOAP}^{3}$.

\section{References}

1. D.-M. Mei et al., Direct detection of MeV-scale dark matter utilizing germanium internal amplification for the charge created by the ionization of impurities. Eur. Phys. J. C 78, 187 (2018)

2. R. Agnese et al., First dark matter constraints from a SuperCDMS single-charge sensitive detector. Phys. Rev. Lett. 121, 051301 (2018)

3. I. Alkhatib et al., (SuperCDMS Collaboration), light dark matter search with a high-resolution athermal phonon detector operated above ground. Phys. Rev. Lett. 127, 061801 (2021)

4. Q. Arnaud et al., (EDELWEISS Collaboration), first germaniumbased constraints on sub-MeV dark matter with the EDELWEISS experiment. Phys. Rev. Lett. 125, 141301 (2020)

5. A.H. Abdelhameed et al., (CRESST Collaboration), first results from the CRESST-III low-mass dark matter program. Phys. Rev. D. 100, 102002 (2019)

6. A. Aguilar-Arevalo et al., (DAMIC Collaboration), results on lowmass weakly interacting massive particles from an $11 \mathrm{~kg} \cdot \mathrm{d}$ target exposure of DAMIC at SNOLAB. Phys. Rev. Lett. 125, 241803 (2020)

7. Michael Crisler et al., (SENSEI Collaboration), SENSEI: first direct-detection constraints on sub-GeV dark matter from a surface run. Phys. Rev. Lett. 121, 061803 (2018) 
8. Orr Abramoff et al., (SENSEI Collaboration), SENSEI: directdetection constraints on sub-GeV dark matter from a shallow underground run using a prototype skipper CCD. Phys. Rev. Lett. 122, 161801 (2019)

9. J.I. Collar, Search for a nonrelativistic component in the spectrum of cosmic rays at Earth. Phys. Rev. D 98, 023005 (2018)

10. P.S. Barbeau, J.I. Collar, O. Tench, Large-mass ultralow noise germanium detectors: performance and applications in neutrino and astroparticle physics. J. Cosmol. Astropart. Phys. 2007(09), 009 (2007)

11. N. Abgrall et al., (Majorana Collaboration), the majorana demonstrator neutrinoless double-beta decay experiment. Adv. High Energy Phys. 2014, 365432 (2014). arXiv:1308.1633

12. K.H. Ackermann et al., (GERDA Collaboration), the GERDA experiment for the search of $0 \nu \beta \beta$ in ${ }^{76} \mathrm{Ge}$. Eur. Phys. J. C 73, 2330 (2013). arXiv:1212.4067

13. P. Barton, M. Amman, R. Martin, K. Vetter, Ultra-low noise mechanically cooled germanium detector. Nucl. Instrum. Methods Phys. Res. A 812, 17-23 (2016)

14. W.-Z. Wei, R. Panth, J. Liu, H. Mei, D.-M. Mei and G.-J. Wang, The impact of the charge barrier height on germanium $(\mathrm{Ge})$ detectors with amorphous-Ge contacts for light dark matter searches. Eur. Phys. J. C 80, 472 (2020). arXiv:2002.04462 [v2]

15. M. Amman, Optimization of amorphous germanium electrical contacts and surface coatings on high purity germanium radiation detectors. arXiv: 1809.03046

16. W.W. Tyler, Deep level impurities in germanium. J. Phys. Chews. Solids 8, 59-65 (1959)

17. A. Castoldi, C. Guazzoni, S. Maffessanti, T. Krings, Germanium drift, detectors: from the idea to the device, in IEEE Nuclear Science Symposium and Medical Imaging Conference Proceedings (NSS/MIC). Sydney, Australia 2018, 1-4 (2018). https://doi.org/ 10.1109/NSSMIC.2018.8824637

18. J. Kemmer et al., Low capacity drift diode. Nucl. Instrum. Methods Phys. Res. A 253, 378-381 (1987)

19. E. Gatti, P. Rehak, J. Kemmer, Proposal for a new tomographic device providing information on the chemical properties of a body section. IEEE Trans. Med. Imaging 5(4), 207-221 (1986). https:// doi.org/10.1109/TMI.1986.4307779
20. Refer to MJD website: https://www.npl.washington.edu/majorana/ design-technologies

21. R. Wittmann, Miniaturization Problems in CMOS Technology: Investigation of Doping Profiles and Reliability, Thesis dissertation (2007)

22. B.S. Neganov, V.N. Trofimov, Colorimetric method measuring ionizing radiation, Otkryt. Izobret. 146, 215 (1985). USSR Patent No. 1037771

23. P.N. Luke, Voltage-assisted calorimetric ionization detector. J. Appl. Phys. 64, 6858 (1988)

24. H. Mei, D.-M. Mei, G.-J. Wang, G. Yang, The impact of neutral impurity concentration on charge drift mobility in germanium. JINST 11, P12021 (2016). arXiv: 1607.03032

25. W.-Z. Wei et al., Investigation of amorphous germanium contact properties with planar detectors made from home-grown germanium crystals. JINST 13, P12026 (2018)

26. Sanford Wagner et al., IEEE standard test procedures for highpurity germanium crystals for radiation detectors, IEEE Std 11601993, pp. 1-36, (1993). https://doi.org/10.1109/IEEESTD.1993. 115139

27. X.-H. Meng et al., Fabrication and characterization of high-purity germanium detectors with amorphous germanium contacts. JINST 14, P02019 (2019)

28. Jianchen Li, Jing Liu, Kyler Kooi, HPGe detector field calculation methods demonstrated with an educational program. GeFiCa. Eur. Phys. J. C 80, 230 (2020)

29. D.-M. Mei et al., Impact of charge trapping on the energy resolution of Ge detectors for rare-event physics searches. J. Phys. G Nucl. Part. Phys., accepted manuscript online 28 May (2020). arXiv:1909.05806

30. Please refer to: http://nucleardata.nuclear.lu.se/toi/nuclide.asp? iZA $=950241$

31. S. Agostinelli et al., Geant4-a simulation toolkit. Nucl. Instrum. Methods Phys. Res. A 506, 250-303 (2003) 